\title{
Aislamiento de ácido úsnico y parietina de Caloplaca saxicola Hoffm.
}

\section{Isolation of the usnic acid and parietin of the Caloplaca saxicola Hoffm.}

\author{
Olivio Castro Mandujano ${ }^{1 *}$, Ana Pastor de Abram ${ }^{1}$, Ingrit E. Collantes Díaz ${ }^{2}$ \\ ${ }^{1 *}$ Pontificia Universidad Católica del Perú, Departamento de Ciencias - Sección Química; Av. Universitaria 1801, San Miguel, \\ Lima 32, Perú. ninocastro2005@hotmail.com \\ ${ }^{2}$ Laboratório de Extração da Universidade Paulista. Av. Paulista 900, Bela vista, C.E.P. 01310-100 São Paulo, Brasil.
}

\section{RESUMEN}

En el presente trabajo se investigó la Caloplaca saxicola (Hoffm.), recolectado cerca de la laguna de Chinchaycocha (Carhuamayo - Cerro de Pasco), a una altitud de $4100 \mathrm{msnm}$. A 240 gramos de muestra, se sometió a una extracción acetónica por maceración; se procedió a separar diversos componentes del extracto, empleando la cromatografía en columna, con el sistema cloroformo-acetona aumentando la cantidad de acetona; luego, las fracciones obtenidas se purifican empleando técnicas cromatográficas y recristalizaciones. Finalmente, se elucidaron y se determinaron las estructuras de los sólidos obtenidos, analizando sus espectros de IR, $\mathrm{RMN} \mathrm{H}^{1}, \mathrm{RMN} \mathrm{C}^{13}$ y EM; éstos son: ácido úsnico y parietina.

Palabras clave: Liquen, Caloplaca saxicola, ácido úsnico, parietina.

\begin{abstract}
In the present work there was investigated the Caloplaca saxicola (Hoffm.), gathered near Chinchaycocha's lagoon (Carhuamayo - Cerro de Pasco), to an altitude of $4100 \mathrm{msnm}$. To 240 grams sample, it surrendered to an extraction acetonic for maceration; one separated diverse components of the extract, using the chromatography in column, with the system chloroform - acetone increasing the quantity of acetone; then, the obtained fractions they purified using chromatographic techniques and recrystallizations Finally, they were elucidated and there decided the structures of the obtained solids, analyzing his spectra of IR, $H^{1} N M R$, $\mathrm{C}^{13} \mathrm{NMR}$, and MS; these are: usnic acid and parietin.
\end{abstract}

Key Words: Lichen, Caloplaca saxicola, usnic acid, parietin.

\section{INTRODUCCIÓN}

El liquen es un organismo simbiótico conformado por un hongo y un alga; se estima que existen aproximadamente 18500 especies; pueden ser encontrados desde las regiones árticas hasta las tropicales, desde planicies hasta regiones montañosas y desde condiciones acuáticas hasta desérticas; desenvolviéndose sobre rocas, corteza, hojas de árboles, suelos, etc. Producen una grande variedad de metabolitos primarios como carbohidratos, aminoácidos y metabolitos secundarios (esteroides, terpenos, cromonas, xantonas, naftoquinonas, antronas, antraquinonas etc.) muchos de ellos son característicos o propios de los líquenes, llamados compuestos liquénicos y son aproximadamente 1050 metabolitos secundarios ya identificados, fueron agrupados de acuerdo al tipo de estructura, éstos son:, dépsidos, depsidonas, ácidos úsnicos, dibenzofuranos ${ }^{2}$.
Recientemente hay un gran interés en el teñido textil con pigmentos naturales por su alta compatibilidad y su no toxicidad. Las antraquinonas son compuestos empleados para estos fines; por ello, actualmente, se están realizando investigaciones en el cultivo de líquenes a partir de hongos ${ }^{3}$. En una investigación de la especie Rumex crispus fue aislado la antraquinona parietina, la cual, indican, tiene actividad antifúngica de 30 a $10 \mu \mathrm{g} / \mathrm{mL}$ que es lo que la hace más eficiente como el fenarimol y el polioxin ${ }^{4}$. El ácido úsnico es un metabolito secundario propio de líquenes presenta actividad inhibidora de tumores; es analgésica, también se usa como conservantes de cremas cosméticas ${ }^{5}$.

El artículo de revisión del género Caloplaca ${ }^{6}$ reporta el estudio de antraquinonas en 253 especies, de las cuales 226 de ellas reportan la presencia de parietina y sólo 5 de ellas presentaban ácido úsnico; 
en este estudio no estaba incluido la especie $C$. saxicola. El objetivo de este trabajo es abordar la identificación de los componentes mayoritarios del liquen C. saxicola porque hasta hoy en día no se encontró estudio fitoquímico de la especie en estudio.

\section{PARTE EXPERIMENTAL}

\section{Colecta de la muestra y la identificación taxonómica.}

El liquen estudiado fue recolectado de las piedras en el distrito de Carhuamayo, provincia Cerro de Pasco, departamento Junín, a una altitud de 4100 msnm, cerca de la laguna de Chinchaycocha. La determinación taxonómica (tabla 1) fue realizada por la Dra. Mónica T. Adler de la Universidad de Buenos Aires-Argentina. C. saxicola (figura 1), pertenece a la familia Teloschisteceae, la cual consiste de 3 pequeños géneros: Teloschistes, Xanthoria y Fulgensia, y además de un género grande, llamado Caloplaca con más de 1000 especies publicadas. El género Caloplaca tiene 6 subgéneros estos son: Pyrenodesmia, Leproplaca, Gasparrinia, Xanthocarpia, Gyalolechia, Caloplaca ${ }^{7}$. La especie C. saxicola Hoffm., es un liquen crustáceo areolado en el centro y hacia los márgenes formando lóbulos anaranjado, saxícola; tiene apotecio circular, plano, sésil, diámetro de $5 \mathrm{~mm}$, con borde talino y epitecio anaranjado. Talo con córtex superior, heterómero con clorobionte Trebouxia sp. Apotecio lecanorino; asca con ocho ascosporas elipsoidales; paráfisis con septos y ramificación en el ápice. Talo $\mathrm{K}+$ (púrpura). Habita en laderas pedregosas y cumbres rocosas ${ }^{8}$.

Tabla 1.- Identificación taxonómica de C. saxicola.

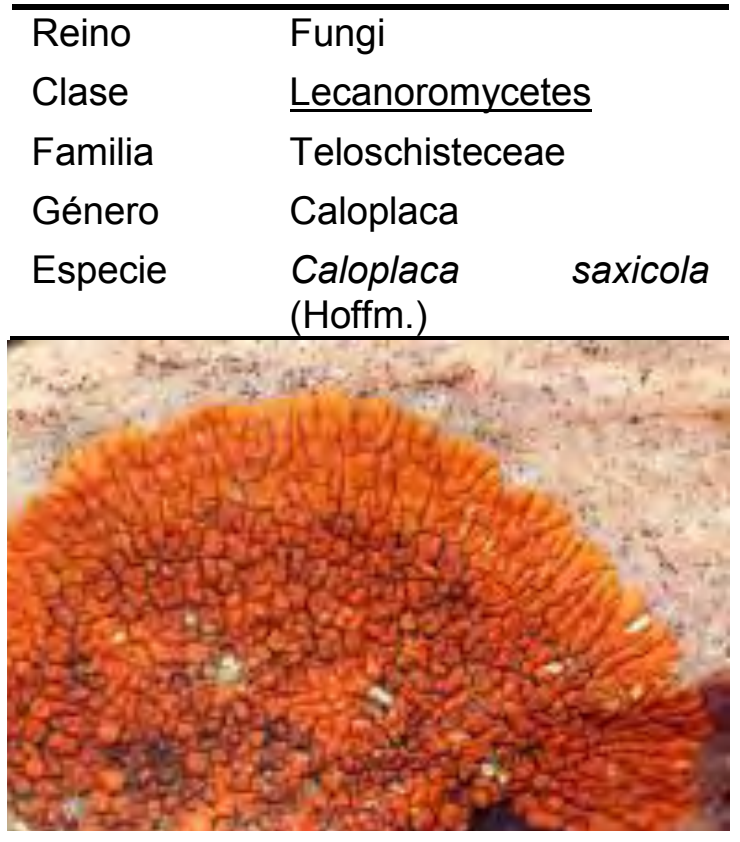

Figura 1.- Liquen C. saxicola.

\section{Aislamiento del ácido úsnico y parietina.}

La muestra limpia, seca y molida (240 g), se sometió a una extracción acetónica por maceración, dos veces; el extracto fue concentrado en rotaevaporador hasta un volumen de $25 \mathrm{~mL}$, luego fue concentrado con nitrógeno hasta sequedad $(2,35$ g), obteniéndose un extracto pastoso, el que fue sometido a cromatografía en columna usando como fase estacionaria sílica gel y como fase móvil la mezcla de solventes cloroformo:acetona, con polaridad creciente en la siguiente proporción: 10:0, $9: 1,8: 2,7: 3,6: 4, \ldots, 1: 9,0: 10$, obteniéndose 80 fracciones, las cuales fueron sometidas a cromatografía en capa delgada (sobre cromatofolios de sílica gel eluyendo en la mezcla de solventes tolueno:acetato de etilo:ácido acético glacial $(6: 4: 1)$ y revelando con una solución de metanol ácido al $10 \%$ con ácido sulfúrico) seguida de calentamiento; fueron reunidas las fracciones desde 23 hasta 34 y desde 65 hasta 72 por separado, porque contenían un solo compuesto cada uno de ellas, las que proporcionaron dos compuestos cristalinos en forma de agujas de color amarillo y naranja.

\section{Determinación del punto de Fusión.}

El punto de fusión del ácido úsnico fue determinado en un aparato Fisher-Johns.

\section{Análisis por Ultravioleta-Visible (UV-Vis).}

Los espectros de UV-Visible fueron registrados en un espectrofotómetro Ultravioleta-Visible, Lambda 2, L.A., Perkin Elmer, el solvente utilizado para diluir la muestra fue etanol de grado para análisis de Merck. Análisis por Infra Rojo (IR).

Los espectros de IR fueron obtenido con el Espectrómetro Infrarrojo, FT - IR, serie 1600, Perkin Elmer, las pastillas de bromuro de potasio $(\mathrm{KBr})$ fueron preparadas en un $3-5 \%$, el $\mathrm{KBr}$ es de grado para análisis de Merck.

\section{Análisis por Cromatografía Líquida de Alta Eficiencia (CLAE).}

Los cromatogramas fueron hechos en un cromatógrafo de marca Kontron HPLC 450 con detector UV 430 y un auto inyector 360, fue usada como fase estacionaria una columna Spherisorb 5 ODS Kontron de $5 \mu \mathrm{m}, 250 \times 4,6 \mathrm{~mm}$ a temperatura de ambiente; fue inyectado $20 \mu \mathrm{L}$ de $0,02 \mathrm{mg} / \mathrm{mL}$ de concentración de muestra solubilizada en acetona, y como fase móvil fue utilizando los solventes: Solvente $\mathrm{A}$, agua bidestilada $1 \% \mathrm{H}_{3} \mathrm{PO}_{4}$ y solvente $\mathrm{B}$, $\mathrm{MeOH} 100 \%$ y la corrida para eluir los compuestos fue $30 \%$ de Sol. B por $1 \mathrm{~min}$., $14 \mathrm{~min}$. después llegó a $70 \%$ del sol. B, 15 min más llegó a $100 \%$ de sol. B por 18 min.; los compuestos fueron detectados a $245 \mathrm{~nm}$. 


\section{Análisis por Resonancia Magnética Nuclear (RMN).}

Los espectros de Resonancia Magnética Nuclear ${ }^{1} \mathrm{H}$ y de ${ }^{13} \mathrm{C}$ fueron registrados en un espectrómetro Varian INOVA DPX-300, operando a 300 y $75 \mathrm{MHz}$ $(7,05 T)$. De acuerdo con la solubilidad de las muestras, los espectros fueron obtenidos en $\mathrm{CDCl}_{3}$, marca Merck.

\section{RESULTADOS Y DISCUSIÓN}<smiles>CC(=O)C1=C(O)C=C(Oc2c([18O])c(O)c(C)c(O)c2C(C)=O)[C@@H](C)C1=O</smiles>

Figura 2.- Estructura molecular del ácido úsnico.

Los cristales con forma de agujas de color amarillo (300 mg) son provenientes de las reunión de las fracciones 23-34 y solubles en $\mathrm{CHCl}_{3}$, poco soluble en $\mathrm{MeOH}, \mathrm{Me}_{2} \mathrm{CO}$, con punto de fusión de 203$204^{\circ} \mathrm{C}$; todos estos datos preliminares nos indica que se trataría del acido úsnico, ver su estructura en la figura 2. Se obtuvo un espectro de UV-Vis en etanol como está representado en la figura 3, comparando este espectro con el de la literatura ${ }^{9}$, presenta similares máximos de absorbancia.

Este compuesto fue inyectado en un CLAE y fue comparado con un patrón de ácido úsnico siguiendo el método establecido en la literatura ${ }^{10}$, la comparación con el patrón indicaba que se trataba del ácido úsnico por la comparación de los tiempos de retención 28,103 min., lo que se comprobó con el análisis de los otros espectros.



Figura 3.- Espectro de UV-Vis del ácido úsnico.
El espectro de RMN ${ }^{1} \mathrm{H}$ (figura 4) presenta señales características de grupos metílicos con desplazamiento químico en 1,76 ppm del C-13; 2,09 ppm del C-16 y 2,67 ppm representa 2 grupos metilos C-15 y C-18, una señal en 5,98 ppm del hidrógeno de $\mathrm{C}-4$ y las señales en 11,02 ppm del hidrógeno $\mathrm{OH}$ del $\mathrm{C}-10 ; 13,31 \mathrm{ppm}$ del hidrógeno $\mathrm{OH}$ del C-8 y 18,84 ppm del hidrógeno del C-3 como está representado en la tabla 2 y comparado con los datos de la literatura, está concordando ${ }^{11,12,13}$.

Tabla 2.- Desplazamiento químico del espectro de $\mathrm{RMN}{ }^{1} \mathrm{H}$ y $\mathrm{RMN}{ }^{13} \mathrm{C}$ del ácido úsnico.

\begin{tabular}{cccccc}
\hline & $\begin{array}{c}\mathbf{H} \\
\mathbf{\delta} \\
(\mathbf{p p m})\end{array}$ & $\begin{array}{c}\mathbf{C} \\
\mathbf{\delta} \\
(\mathbf{p p m})\end{array}$ & $\begin{array}{c}\mathbf{H} \\
\mathbf{\delta} \\
(\mathbf{p p m})\end{array}$ & $\begin{array}{c}\mathbf{C} \\
\mathbf{\delta} \\
(\mathbf{p p m})\end{array}$ \\
\hline $\mathbf{1}$ & - & 198,02 & $\mathbf{1 0}$ & 11,02 & 163,85 \\
& & & & $s$ & \\
$\mathbf{2}$ & - & 179,33 & $\mathbf{1 1}$ & - & 105,19 \\
$\mathbf{3}$ & 18,84 & 155,17 & $\mathbf{1 2}$ & - & 59,04 \\
& $s$ & & & & \\
$\mathbf{4}$ & $5,98 s$ & 98,29 & $\mathbf{1 3}$ & $1,76 s$ & 27,87 \\
$\mathbf{5}$ & - & 101,49 & $\mathbf{1 4}$ & - & 200,29 \\
$\mathbf{6}$ & - & 191,68 & $\mathbf{1 5}$ & $2,67 s$ & 32,09 \\
$\mathbf{7}$ & - & 109,28 & $\mathbf{1 6}$ & $2,09 s$ & 7,51 \\
$\mathbf{8}$ & 13,31 & 157,47 & $\mathbf{1 7}$ & - & 201,75 \\
& $s$ & & & & \\
$\mathbf{9}$ & & 103,92 & $\mathbf{1 8}$ & $2,67 s$ & 31,25 \\
\hline
\end{tabular}

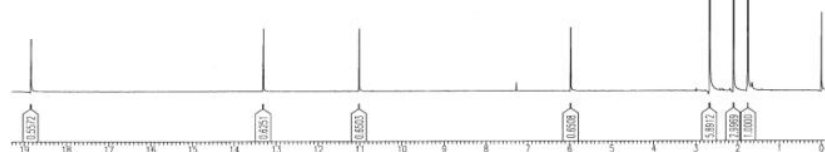

Figura 4.- Espectros de $\mathrm{RMN}{ }^{1} \mathrm{H}$ del ácido úsnico en $\mathrm{CDCl}_{3}$ a $300 \mathrm{MHz}$

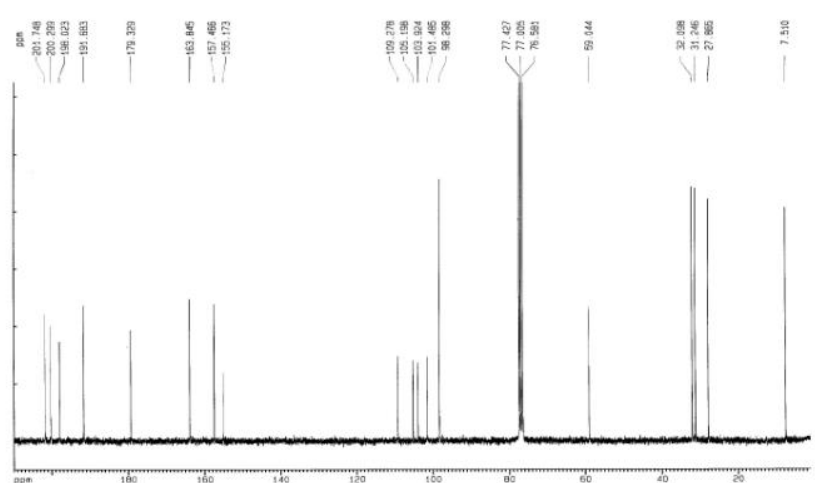


Figura 5.- Espectros de $\mathrm{RMN}{ }^{13} \mathrm{C}$ del ácido úsnico en $\mathrm{CDCl}_{3}$ a $75 \mathrm{MHz}$.

El espectro de $\mathrm{RMN}{ }^{13} \mathrm{C}$ (figura 5 ) presenta 18 carbonos, con señales de grupos metilos, siendo uno de ellos bien protegido en campo alto con desplazamiento químico de 7,51 ppm de C-16 y las señales 27,87 del C-13, 31,25 del C-18, 32,09 ppm del C-15; un pico en 59,04 ppm característicos de carbono cuaternario C-12; las señales de los grupos carbonilos en 198,02 ppm C-1; 200,29 ppm C-14 y 201,75 ppm C-17, como mostramos en la tabla 2, la comparación de estas señales con el ácido úsnico disponibles en la literatura ${ }^{11,1213}$, permitió verificar que se trataba del mismo compuestos.<smiles>COc1cc(O)c2c(c1)C(=O)c1cc(C)cc(O)c1C2=O</smiles>

Figura 6.- Estructura molecular de parietina.

Los cristales con forma de agujas de color anaranjado $(126 \mathrm{mg})$ son provenientes de la reunión de las fracciones 65-72, solubles en $\mathrm{CHCl}_{3}, \mathrm{MeOH}$ y $\mathrm{Me}_{2} \mathrm{CO}$; de los cristales se obtuvo un espectro de UV-Vis (figura 6 ); fue tomado en etanol $(0,01 \%)$; los valores de absorbancia máxima observados son 223; 251; 264; 287 y $433 \mathrm{~nm}$ y confrontando estos valores con los de la literatura ${ }^{14}$ de antraquinonas 1,5-dihidroxiantraquinona 253; 276; 287 y $330 \mathrm{~nm}$ verificamos que en este caso el mismo esqueleto; presentan semejantes valores de absorbancia máxima y cuando comparado con el espectro de la parietina de la literatura ${ }^{15}$ observamos que es idéntico al espectro de la figura 7.

Este compuesto fue inyectado en un CLAE (el cromatograma obtenido es la figura 8) y fue comparado con un patrón de parietina siguiendo el método establecido por la literatura ${ }^{10}$; la comparación de los tiempos de retención $(31,732 \mathrm{~min})$ con el patrón indicaba que se trataba de parietina; lo que se comprobó con el análisis de los otros espectros.

El espectro IR (en $\mathrm{KBr}$ ) de la figura 8 se observan bandas de absorción como el de $3438 \mathrm{~cm}^{-1}$ lo cual nos indica presencia de grupos $\mathrm{OH}$, a $3035 \mathrm{~cm}^{-1}$ vibración del $\mathrm{C}-\mathrm{H}$ aromático, a $2921 \mathrm{~cm}^{-1}$ vibración asimétrico del grupo $\mathrm{CH}_{3}$, a $2844 \mathrm{~cm}^{-1}$ presencia de la vibración simétrica del grupo $\mathrm{CH}_{3}$, a $1691 \mathrm{~cm}^{-1}$ nos indica un alargamiento del $\mathrm{C}=0 ; 1630 \mathrm{~cm}^{-1}$ para el grupo $\mathrm{C}=\mathrm{C}$ conjugado; $1295 \mathrm{~cm}^{-1}$ y 1035 alargamiento del C-O-C simétrico y antisimétrico, concordante con la literatura ${ }^{16}$.

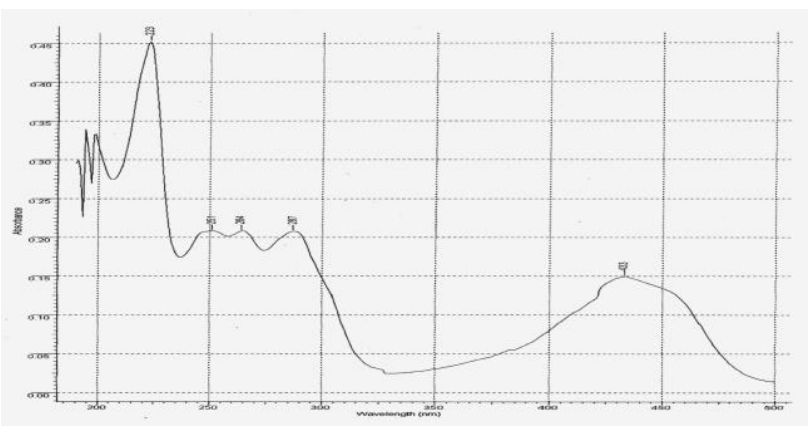

Figura 7.- Espectro de UV-Visible de parietina en etanol $(0,01 \%)$.

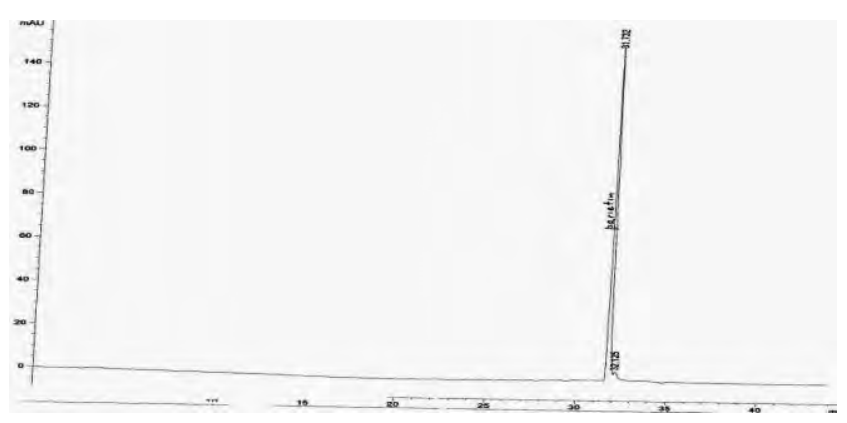

Figura 8.- Cromatograma de CLAE de parietina.

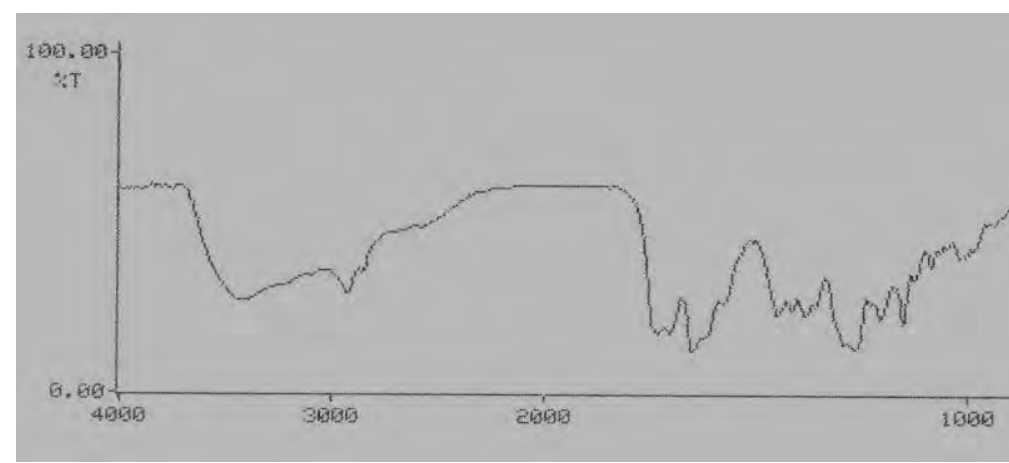

Figura 9.- Espectro de Infra rojo de parietina.

En el espectro RMN ${ }^{1} \mathrm{H}$ (figura 10) se observa una señal característica del grupo metil $\mathrm{CH}_{3}$ en $2,45 \mathrm{ppm}$ singlete $(s)$; hay una sola señal $(s)$, característico de metoxilos $\left(\mathrm{OCH}_{3}\right)$ a $3,94 \mathrm{ppm}$; también se observa la presencia de 4 hidrógenos aromáticos a $6,68 \mathrm{ppm} \mathbf{H}$ de $\mathrm{C}$-10 duplete $(d)$ con constante de acoplamiento (J) de 2,4 Hz; 7,08 ppm $\mathbf{H}$ de C-2 $d$ con $J$ de $1,5 \mathrm{~Hz}$; $7,36 \mathrm{ppm} \mathbf{H}$ de $\mathrm{C}-8 d$ con $J$ de $2,4 \mathrm{~Hz}$ y $7,62 \mathrm{ppm} \mathbf{H}$ de $\mathrm{C}-4 d$ con $J$ de $1,2 \mathrm{~Hz}$; estos 4 hidrógenos presentan multiplicidad de hidrógenos acoplando en meta y dos singletes, correspondiente al grupo $\mathrm{OH}$ en 12,12 ppm del C-1 y 12,31 ppm del C-11 como mostramos en la tabla 3 donde se observaron los 
hidrógenos con sus respectivas multiplicidades; la comparación de estas señales con parietina disponibles en la literatura ${ }^{16,17,18}$, permitió verificar que se trataba del mismo compuesto.

Tabla 3.- Desplazamiento químico del espectro de RMN ${ }^{1} \mathrm{H}$ y $\mathrm{RMN}{ }^{13} \mathrm{C}$ de parietina.

\begin{tabular}{cccc}
\hline & $\begin{array}{c}\mathbf{H} \\
\mathbf{\delta}(\mathbf{p p m})\end{array}$ & $\begin{array}{c}\mathbf{C} \\
\mathbf{\delta}(\mathbf{p p m})\end{array}$ & $\mathbf{D E P T}$ \\
\hline $\mathbf{1}$ & - & 162,51 & $\mathrm{C}$ \\
$\mathbf{2}$ & $7,08 d$, & 124,49 & $\mathrm{CH}$ \\
& 1,5 & & \\
$\mathbf{3}$ & - & 148,43 & $\mathrm{C}$ \\
$\mathbf{4}$ & $7,62 d$, & 121,26 & $\mathrm{CH}$ \\
& 1,2 & & \\
$\mathbf{5}$ & $7,36 d$, & 133,22 & $\mathrm{C}$ \\
& 2,4 & & \\
$\mathbf{6}$ & - & 181,99 & $\mathrm{C}$ \\
$\mathbf{7}$ & $6,68 d$, & 135,26 & $\mathrm{C}$ \\
& 2,4 & & \\
$\mathbf{8}$ & - & 108,19 & $\mathrm{CH}$ \\
$\mathbf{9}$ & - & 166,56 & $\mathrm{C}$ \\
$\mathbf{1 0}$ & - & 106,77 & $\mathrm{CH}$ \\
$\mathbf{1 1}$ & - & 165,19 & $\mathrm{C}$ \\
$\mathbf{1 2}$ & - & 110,27 & $\mathrm{C}$ \\
$\mathbf{1 3}$ & - & 190,79 & $\mathrm{C}$ \\
$\mathbf{1 4}$ & - & 113,68 & $\mathrm{C}$ \\
$\mathbf{1 5}$ & $2,45 s$ & 22,13 & $\mathrm{CH}_{3}$ \\
$\mathbf{1 6}$ & $3,94 \mathrm{~s}$ & 56,06 & $\mathrm{OCH}_{3}$ \\
$\mathrm{OH}-$ & $12,12 s$ & - & - \\
$\mathrm{C}-1$ & & & \\
$\mathrm{OH}-$ & $12,31 s$ & - & - \\
$\mathrm{C}-11$ & & & \\
\hline
\end{tabular}

El espectro de RMN ${ }^{13} \mathrm{C}$ (figura 11) presenta 16 carbonos, una señal de grupo metilo en $22,13 \mathrm{ppm}$ C-15, un pico en 56,06 ppm característico de metoxilo C-16; 190,79 ppm C-12 y 181,99 ppm C-6 característico de grupos carbonilos y 12 picos que están entre 167 y 106 ppm característicos de carbonos en la región aromática. Ya el espectro de DEPT (figura 12) nos proporciona los datos de los carbonos que están enlazados directamente con hidrógenos, con el que confirmamos la propuesta del grupo metilo y del metoxilo; también observamos que solo 4 de los carbonos de la región aromática están enlazados a hidrógenos y son 10 carbonos cuaternarios; los desplazamientos químicos 124,49; 121,$26 ; \quad 133,22 ; \quad 135,26 \quad$ ppm son los correspondientes carbonos $\mathrm{CH}$ y como explicamos arriba los desplazamientos químicos 22,13 y 56,06 ppm son los correspondientes a $\mathrm{CH}_{3}$; las señales en 162,$51 ; 148,43 ; 133,22$; 181,99; 135,26; 166,56;
165,19 ppm son los correspondientes a los carbonos cuaternarios la comparación de estas señales con parietina disponibles en la literatura ${ }^{18}$, permitió verificar que se trataba de la antraquinona parietina, también llamada de fiscion (1,8-dihidroxi-6-metoxi-3metil-9,10-antracenediona).

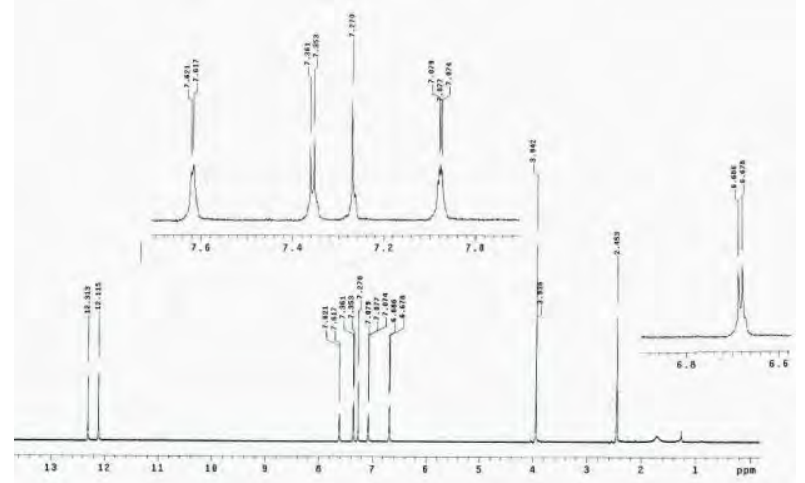

Figura 10.- Espectro de $\mathrm{RMN}{ }^{1} \mathrm{H}$ de parietina en $\mathrm{CDCl}_{3}$ a $300 \mathrm{MHz}$.

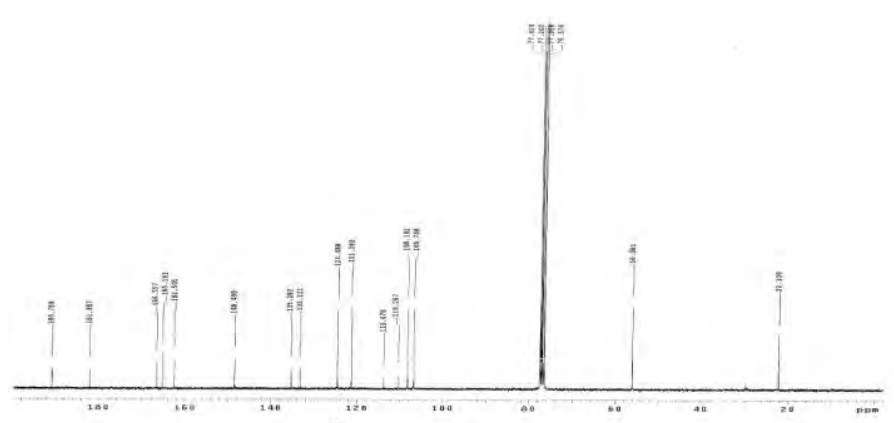

Figura 11: Espectro de $\mathrm{RMN}{ }^{13} \mathrm{C}$ de parietina en $\mathrm{CDCl}_{3} 75 \mathrm{MHz}$.

Figura 12: Espectro de RMN ${ }^{13} \mathrm{C}$ - DEPT de parietina.

\section{CONCLUSIONES}

Anteriormente el liquen Caloplaca saxicola Hoffm. no presenta ningún estudio fitoquímico y es, en este artículo que presentamos el aislamiento e identificación por técnicas espectroscópicas de los compuestos mayoritarios presentes: el ácido úsnico (300mg) y la antraquinona parietina (126mg), son la primera vez reportados en la especie. 
El aislamiento tuvo una eficiencia de $12,77 \%$ para el ácido úsnico y 5,36\% para la antraquinona parietina.

\section{AGRADECIMIENTOS}

Esta investigación fue realizada gracias a la colaboración de la Dra. Mónica T. Adler, de la Universidad de Buenos Aires - Argentina, por la identificación botánica del liquen estudiado y al Dr. John Elix de la Universidad Nacional de Australia por los análisis de HPLC.

\section{BIBLIOGRAFÍA}

1. Molnár, K.; Farkas, E, Current Results on Biological Activities of Lichen Secondary Metabolites: a Review, Zeitschrift fur Naturforsch C, (2010), 65c, 157-173.

2. Honda, N.K.; Vilegas, W.; A Química dos Liquenes, Química Nova, (1998), 21, 110 124.

3. Nagia, F.A.; El-Mohamedy, R.S.R., Dyeing of Wool with Natural Anthraquinone Dyes from Fusarium oxysporum, Dyes and Pigments, (2007), 75, 550-555.

4. Choi, G.J.; Lee, S.W.; Jang, K.S.; Kim, J.S.; Cho, K.Y.; Kim, J.C.; Effects of Chrysophanol, parietin, and nepodin of Rumex crispus on Barley and Cucumber Powdery Mildews; Crop Protection, (2004), 23, 1215-1221.

5. Huneck, S.; The Significance of Lichens and their Metabolites; Naturwissenschaften, (1999), 86, 559-570.

6. Santesson, J.; Anthraquinones in Caloplaca; Phytochemistry, (1970), 9, 2149-2166.

7. Sochting, U.; Lutzoni, F.; Molecular Phylogenetic Study at the Generic Boundary between the Lichen-Forming Fungi Caloplaca and Xanthoria (Ascomycota, Teloschistaceae); Mycological Research, (2003), 107(11), 1266-1276.

8. Ramírez, A.; Cano, A.; Líquenes de Pueblo Libre, una localidad andina en la Cordillera Negra (Huaylas, Ancash, Perú); Revista Peruana de Biología, (2005), 12(3), 383- 396.

9. Stocker-Wörgötter, E.; Elix, J.A.; Grube, M.; Secondary Chemistry of Lichen-forming Fungi: Chemosyndromic Variation and DNAanalyses of Cultures and Chemotypes in the Ramalina farinaceae Complex; The Bryologist, (2004), 107(2), 152-162.

10. Feige, G.B.; Lumbsch, H.T.; Huneck, S. ; Elix, J.A. ; Identification of Lichen Substances by a Standardized High-performance Liquic Chromatographic Method, Journal of Chromatography, (1993), 646, 417-427.
11. Rashid, M.A.; Majid, M.A.; Quader, M.A.; Complete NMR Assignments of (+)-usnic acid, Fitoterapia, (1999), 70, 113-115.

12. König, G.M.; Wright, A.D.; ${ }^{1} \mathrm{H}$ and ${ }^{13} \mathrm{C}-\mathrm{NMR}$ and Biological Activity Investigations of Four Lichen-derived Compounds; Phytochemical Analysis; (1999), 10, 279-284.

13. Castro Mandujano, O.N.; Aislamiento del ácido úsnico de Flavoparmelia caperata y su determinación cuantitativa por Espectroscopia UV, en Diez Líquenes; Revista de la Sociedad Química del Perú; en proceso de publicación, (2010).

14. Scott A.I., Interpretation of the Ultraviolet Spectra of Natural Products, The Macmillan Company, (1964), pag288.

15. Chu, X.; Sun, A.; Liu, R.; Preparative Isolation and Purification of Five Compounds from the Chinese Medicinal Herb Polygonum cuspidatum Sieb. Et Zucc by High-speed Counter-current Chromatography; Journal of Chromatography A, (2005), 1097, 33-39.

16. Edwards, H.G.M.; Newton, E.M.; WynnWilliams, D.D.; Coombes, S.R.; Molecular Spectroscopic Studies of Lichen Substances 1: Parietin and Emodin; Journal of Molecular Structure; (2003), 648, 49-59.

17. Manojlovic, I.; Bogdanovic-Dusanovic, G.; Gritsanapan, W.; Manojlovic, N.; Isolation and Identification of Anthraquinones of Caloplaca cerina and Cassia tora*; Chemical Paper, (2006), 60(6), 466-468.

18. Smetanina, O.F.; Kalinovskii, A.I.; Khudyakova, Y.V.; Slinkina, N.N.; Pivkin, M.V.; Kuznetsova, T.A.; Metabolites from the Marine Fungus Eurotium repens; Chemistry of Natural Compounds, (2007), 43(4), 395398 